\title{
Quantum instability and edge entanglement in the quasi-long-range order
}

\author{
W. Son, ${ }^{1,2}$ L. Amico, ${ }^{3,4}$ F. Plastina, ${ }^{5}$ and V. Vedral ${ }^{1,2,6}$ \\ ${ }^{1}$ The School of Physics and Astronomy, University of Leeds, Leeds, LS2 9JT, United Kingdom \\ ${ }^{2}$ Centre for Quantum Technologies, National University of Singapore, 3 Science Drive 2, Singapore 117543 \\ ${ }^{3}$ Departemento de Fsica de Materiales, Universitad Complutense de Madrid, 28040 Madrid, Spain \\ ${ }^{4}$ MATIS-INFM \& Dipartimento di Metodologie Fisiche e Chimiche (DMFCI), \\ Università di Catania, viale A. Doria 6, 95125 Catania, Italy \\ ${ }^{5}$ Dip. Fisica, Università della Calabria, 8 INFN - Gruppo collegato di Cosenza, 87036 Arcavacata di Rende (CS) Italy \\ ${ }^{6}$ Department of Physics, National University of Singapore, 2 Science Drive 3, Singapore 117542
}

(Dated: November 3, 2018)

\begin{abstract}
We investigate the build-up of quasi-long-range order in the $X X$ chain with transverse magnetic field at finite size. As the field is varied, the ground state of the system displays multiple level crossings producing a sequence of entanglement jumps. Using the partial fidelity and susceptibility, we study the transition to the thermodynamic limit and argue that the topological order can be described in terms of kink-antikink pairs and marked by edge spin entanglement.

PACS numbers: 03.67.-a, 03.67.Mn, 42.50.Lc, 73.43.Nq, 75.10.Pq, 75.45.+j
\end{abstract}

Strongly interacting many-body systems display a variety of zero temperature Quantum Phase Transitions (QPT) [1]. Quantum criticality is a property of the ground state of the system, ultimately arising from a reshuffling of the system's energy spectrum when control parameters are varied. In many cases, this is accompanied by a symmetry breaking and by the appearance of an order parameter indicating that the macroscopic order is reached, characterized by long range correlations. There also exist phase transitions with vanishing local order parameter. A typical example is the BerezinskiiKosterlitz-Thouless (BKT) transition, occurring, e.g. in a system of planar classical spins at a finite temperature [2]. The transition is characterized by a 'distortion' of topological nature of the spatial spin configuration, giving rise to quasi-long-range order in the system, whose correlation functions display a power-law decay [3]. In the present paper we will be dealing with ground states of one dimensional systems displaying topological order. The topological order in one dimension is peculiar and it can be characterized by a sensitivity of the ground state by varying the boundary conditions, giving rise to certain solitonic edge states (see for instance [4]).

The prospect of practical applications, such as quantum information processing, has led to an intense activity aimed at a direct inspection of the properties of the ground state of a given system. Our purpose is to characterize ground states with a non trivial topological order through their entanglement content [5]. To illustrate what we believe are generic features of topologically ordered states with vanishing local order parameter, we focus on a specific system: the spin $1 / 2 \mathrm{XX}$ chain in external magnetic field. According to the general equivalence between the classical- $d+1$ and quantum- $d$ criticality [6], this model displays a QPT from a polarized to a BKT phase with quasilong range order. For such a model, we demonstrate that the quasi-long range or- der manifests itself in the formation of 'entangled edge states' (with spins at the edge of the chain sharing entanglement quite differently from bulk spins) and that it is characterized at finite size by an instability of the ground state determined by a sequence of energy level crossings as the magnetic field is varied [7]. This gives rise to sudden jumps of both the fidelity [8] and the pairwise entanglement (which, thus, behave non-analytically even at finite size). As the size of the chain increases, the number of crossings grows until they become dense within a sharply defined critical region. At the same time, their effect weakens, and the behavior of an infinite chain can be obtained through a smearing of the finite size observables, a procedure that we apply to the partial state fidelity. Again, however, this holds true only for bulk spins, while those at the boundaries show a kind of rigidity and a reduced sensitivity to the QPT.

\section{THE GROUND STATE OF THE XX MODEL}

We consider $N$ spin $1 / 2$ particles on a line, coupled by nearest neighbor XX interaction, with Hamiltonian

$$
H=-J\left[\sum_{i=1}^{N} \frac{1}{2}\left(\sigma_{i}^{x} \sigma_{i+1}^{x}+\sigma_{i}^{y} \sigma_{i+1}^{y}\right)+B \sigma_{i}^{z}\right]
$$

where the exchange constant $J$ has been taken as the energy unit. In the thermodynamic limit, the system undergoes a first order transition from a fully polarized to a critical phase with quasi-long range order [3, 9].

Assuming open boundaries (with $\sigma_{N+1}=0$ ), we employ the Jordan-Wigner and Fourier transformations to introduce the fermion operators

$$
d_{k}=\sqrt{\frac{2}{N+1}} \sum_{l=1}^{N} \sin \left(\frac{\pi k l}{N+1}\right) \bigotimes_{m=1}^{l-1} \sigma_{m}^{z} \sigma_{l}^{-},
$$


that diagonalize $H: H=\sum_{k=1}^{N} \Lambda_{k} d_{k}^{\dagger} d_{k}+N B \mathbb{1}$ with $\Lambda_{k}=-2 B+2 \cos [(\pi k) /(N+1)]$. The $2^{N}$ eigenenergies and eigenkets are $\epsilon_{i} \equiv \sum_{k=1}^{N} \Lambda_{k} \alpha_{k}^{(i)}+N B$, and $\left|\psi_{i}\right\rangle=$ $\Pi_{k=1}^{N}\left(d_{k}^{\dagger}\right)_{k}^{(i)}|\Omega\rangle$, with $\alpha_{k}^{(i)}=\left\langle\psi_{i}\left|d_{k}^{\dagger} d_{k}\right| \psi_{i}\right\rangle \in\{0,1\}$. The state $|\Omega\rangle$ is the fermion vacuum: $d_{k}|\Omega\rangle=0 \forall k$.

The ground state and its energy vary with $B$, and different ground states can be classified in terms of the number of level crossings occurring in the system as $B$ changes. Specifically, when $B>1, \Lambda_{k}<0$ for any $k$ and, thus, the lowest eigenvalue is obtained by taking the state with $\alpha_{k}=1 \forall k$. The ground state energy remains $\epsilon_{g}^{0}=-N B$ as long as $B>\cos [\pi /(N+1)]$. For these values of $B$ no level crossing occurs; the ground state is $\left|\psi_{g}^{0}\right\rangle=\prod_{l=1}^{N} d_{l}^{\dagger}|\Omega\rangle$. The first crossing occurs at $B=\cos [\pi /(N+1)] \equiv B_{1}$. For $\cos [2 \pi /(N+1)]<$ $B<\cos [\pi /(N+1)]$ all of the $\Lambda_{k}$ are negative except for $\Lambda_{1}$. Thus, the ground state energy is obtained by subtracting its positive contribution: $\epsilon_{g}^{1}=\epsilon_{g}^{0}-\Lambda_{1}$. The corresponding eigenstate is $\left|\psi_{g}^{1}\right\rangle=d_{1}\left|\psi_{g}^{0}\right\rangle$. Letting $B_{k}=\cos [k \pi /(N+1)]$ and defining the $k$-th region, $B_{k+1}<B<B_{k}$, we can iterate the procedure above to find the ground state $\left|\psi_{g}^{k}\right\rangle=d_{k} d_{k-1} \cdots d_{1}\left|\psi_{g}^{0}\right\rangle=$ $\prod_{l=k+1}^{N} d_{l}^{\dagger}|\Omega\rangle$ and its energy as

$$
\epsilon_{g}^{k}=-(N-2 k) B-2 \sum_{l=1}^{k} \cos \left(\frac{\pi l}{N+1}\right)
$$

where $k$ represents the number of crossings, $1 \leq k \leq N$. For $B<B_{N}$, no other intersection occurs and the ground state is simply given by the fermion vacuum state. The energy crossings are plotted vs $B$ in Fig. 2 (a).

To investigate the structure of the ground state and its entanglement content, we re-write it in the spin language, using the eigenstates of $\sigma_{i}^{z}$. For $k=0$, the ground state is $\left|\varphi_{g}^{0}\right\rangle=|\uparrow\rangle^{\otimes N}$, which is separable. After the 1-st crossing, it becomes $\left|\varphi_{g}^{1}\right\rangle=\left[\sum_{l=1}^{N} S_{l}^{1}\left(\prod_{m=1}^{l-1} \sigma_{m}^{z} \sigma_{l}^{-}\right)\right]\left|\varphi_{g}^{0}\right\rangle$, where $S_{l}^{k} \equiv \sqrt{2 /(N+1)} \sin [(\pi k l) /(N+1)]$. This is an entangled state, given by a symmetric superposition of all possible kets with one flipped spin. After $k$ level crossings, the ground state is given by $\left|\varphi_{g}^{k}\right\rangle=$ $\prod_{k^{\prime}=1}^{k}\left[\sum_{l=1}^{N} S_{l}^{k^{\prime}}\left(\prod_{m=1}^{l-1} \sigma_{m}^{z} \sigma_{l}^{-}\right)\right]|\uparrow\rangle^{\otimes N}$. Explicitly

$$
\left|\varphi_{g}^{k}\right\rangle=\sum_{l_{1}<l_{2}<\cdots<l_{k}} C_{l_{1} l_{2} \cdots l_{k}}\left|l_{1}, l_{2}, \cdots, l_{k}\right\rangle
$$

where $\left|l_{1}, l_{2}, \cdots, l_{k}\right\rangle$ is the state with flipped spins at sites $l_{1}, l_{2}, \cdots, l_{k}$, while the amplitudes are given by $C_{l_{1} l_{2} \cdots l_{k}}=\sum_{P}(-1)^{P} S_{l_{1}}^{P(1)} S_{l_{2}}^{P(2)} \cdots S_{l_{3}}^{P(3)}$, where the sum extends over the permutation group. At each crossing point, the ground state jumps discontinuously in the spin Hilbert space from one symmetric subspace to another, orthogonal to the previous one. (a)
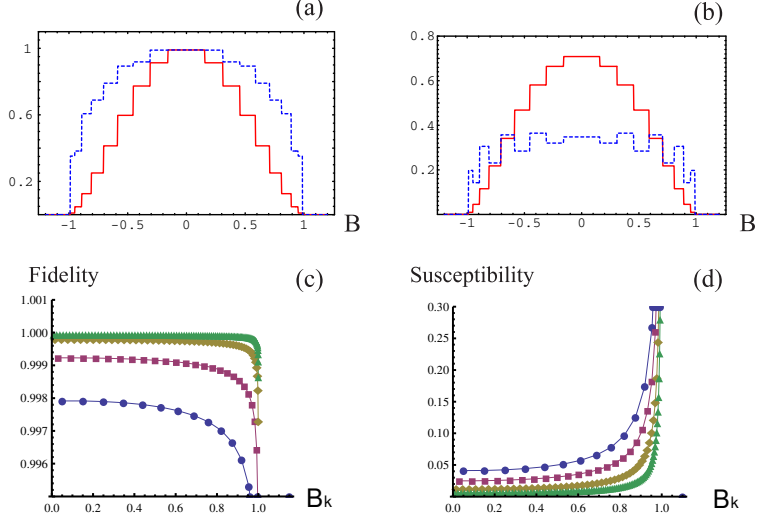

FIG. 1: (color online) Comparison of the entanglement shared by spins at the beginning and at the center of the chain.(a) One-tangle for spin at site $l$ as a function of the magnetic field for $l=1$ (solid line) and $l=9$ (dotted). (b) Nearestneighbor concurrence between spins 1 and 2 (solid line), and 9 and 10 (dotted). All of the plots are for $N=19$. (c) Partial state fidelities and (d) Partial state fidelity susceptibilities as a function of external magnetic field $B_{k}=\cos \left(\frac{\pi k}{N+1}\right)$ for $N=30$ (blue), $N=50$ (red), $N=100$ (yellow) and $N=200$ (green) at $l=(N+1) / 2$. The fidelity approaches 1 as $N$ increases. The parameters in the figures are dimensionless.

\section{FINITE SIZE EFFECTS}

We now study ground state correlation, entanglement and fidelity at finite size. Due to the symmetry of the overall state, the one-spin reduced density matrix is purely diagonal, $\rho_{l}=\frac{1}{2} \operatorname{diag}\left(1+\left\langle\sigma_{l}^{z}\right\rangle, 1-\left\langle\sigma_{l}^{z}\right\rangle\right)$; while for two spins at sites $(l, m)$, one has $\rho_{l m}=a_{+}|\uparrow \uparrow\rangle\langle\uparrow \uparrow$ $\left.\left|+a_{-}\right| \downarrow \downarrow\right\rangle\left\langle\downarrow \downarrow\left|+b_{+}\right| \uparrow \downarrow\right\rangle\left\langle\uparrow \downarrow\left|+b_{-}\right| \downarrow \uparrow\right\rangle\langle\downarrow \uparrow|+e(|\uparrow \downarrow\rangle\langle\downarrow \uparrow$ $|+| \downarrow \uparrow\rangle\langle\uparrow \downarrow|)$, with $a_{ \pm}=\frac{1}{4}\left[1 \pm\left\langle\sigma_{l}^{z}\right\rangle \pm\left\langle\sigma_{m}^{z}\right\rangle+\left\langle\sigma_{l}^{z} \sigma_{m}^{z}\right\rangle\right], b_{ \pm}=$ $\frac{1}{4}\left[1 \pm\left\langle\sigma_{l}^{z}\right\rangle \mp\left\langle\sigma_{m}^{z}\right\rangle-\left\langle\sigma_{l}^{z} \sigma_{m}^{z}\right\rangle\right]$ and $e=\frac{1}{2}\left\langle\sigma_{l}^{x} \sigma_{m}^{x}\right\rangle$. Here, the local magnetization and transverse correlation are given by $\left\langle\sigma_{l}^{z}\right\rangle=1-g_{l, l}$ and $\left\langle\sigma_{l}^{z} \sigma_{m}^{z}\right\rangle=\left(1-g_{l, l}\right)\left(1-g_{m, m}\right)-g_{l, m}^{2}$, where

$$
g_{l, m}=2 \sum_{r=1}^{k} S_{l}^{r} S_{m}^{r}=\frac{S_{l}^{k+1} S_{m}^{k}-S_{l}^{k} S_{m}^{k+1}}{2\left[\cos \left(\frac{\pi l}{N+1}\right)-\cos \left(\frac{\pi m}{N+1}\right)\right]},
$$

that depends on the field $B$ through the index $k$. The longitudinal correlation function $\left\langle\sigma_{l}^{x} \sigma_{m}^{x}\right\rangle$ is [10]:

$$
\left\langle\sigma_{l}^{x} \sigma_{m}^{x}\right\rangle=\left|\begin{array}{cccc}
G_{l, l+1} & G_{l, l+2} & \ldots & G_{l, m} \\
G_{l+1, l+1} & G_{l+1, l+2} & \ldots & G_{l+1, m} \\
\vdots & \vdots & \ddots & \\
G_{m-1, l+1} & G_{m-1, l+2} & \ldots & G_{m-1, m}
\end{array}\right|
$$

where $G_{l, m}=\delta_{l, m}-g_{l, m}$. This determinant becomes of the Toeplitz type in the thermodynamic limit. For nearest neighbors, we simply get $\left\langle\sigma_{l}^{x} \sigma_{l+1}^{x}\right\rangle=G_{l, l+1}$. 
With these density matrices at hand, we discuss the entanglement encoded in the state. Entanglement between a single spin and the rest of the chain can be measured by the one-tangle $\tau_{l}=1-\left\langle\sigma_{l}^{z}\right\rangle^{2}$ 11]. This quantity depends on the site for finite chain, and, as a function of $B$, it displays jumps at each crossing point $B_{k}$. Specifically: (i) $\tau_{l}$ equals one at zero field for every site; (ii) the jumps near $B= \pm 1$ become higher and higher moving from the end points towards the center of the chain, see Fig. 1 (a). Thus, at the onset of the critical region, bulk spins are more entangled than end ones.

To evaluate pairwise entanglement, we use the concurrence [1],$C_{l, m}=2 \max \left\{0,|e|-\sqrt{a_{+} a_{-}}\right\}$. Its behavior as a function of $B$ for nearest neighboring spins with jumps at crossing points is shown in Fig. 1(b). In particular, around $B=0$, the pairwise entanglement is bigger near the end points of the chain; while the reverse occurs at the border of the critical region.

The QPT can be further analyzed through the quantum fidelity of ground states with slightly different fields, $B$ and $\tilde{B}=B+\delta B$. Specifically, we consider the partial state fidelity of reduced density matrix $\rho_{a}(B)=\operatorname{Tr}_{b} \rho(B)$ when the system is partitioned as $a+b: F_{a}(B, \tilde{B})=$ $\operatorname{Tr} \sqrt{\sqrt{\rho_{a}(B)} \rho_{a}(\tilde{B}) \sqrt{\rho_{a}(B)}} . \quad F$ characterizes the degree of change of the state as the field is varied. For our purposes, it is sufficient to consider the subsystem $a$ to consist of just the $l$-th spin 12]. Within the critical region, $F_{l}$ is unit everywhere except for a series of discrete and sharp drops at the crossing points $B=B_{k}$. For large system sizes, when the crossings are dense in the interval $|B| \leq 1$, we can perform a coarse-graining and evaluate $F$ only at these points. As a result of this procedure, only the drop at $B=1$ remains, while all of the intermediate ones are smeared out, see Fig. 1(c). Interestingly enough, this behavior occurs only for bulk spins. If, instead, one of the end spins is singled out, the coarse grained fidelity stays flat.

An even more direct evidence that for large $N$ the state of the bulk spins changes essentially in a continuous way except for the critical point $B=1$, is obtained by looking at the fidelity susceptibility [12]: $\chi_{F}^{l}=$ $\lim _{\delta B \rightarrow 0}-2 \ln F_{l} /(\delta B)^{2}$, plotted for a bulk spin in Fig [1 (b). This behavior appears related to the essential singularity shown by the block entropy at $B=1[13]$.

\section{INFINITE SPIN CHAIN}

For $N \rightarrow \infty$, the intervals $B_{k+1}<B<B_{k}$ become infinitesimally small and $\omega \equiv(\pi k) /(N+1)$ becomes continuous, $\omega \approx \arccos (B)$, so that the sum in (3) gives an energy per spin

$$
\lim _{N \rightarrow \infty} \frac{\epsilon_{g}(B)}{N}=\frac{2}{\pi}\left[B\left(\arccos B-\frac{\pi}{2}\right)-\sqrt{1-B^{2}}\right],
$$

(a)

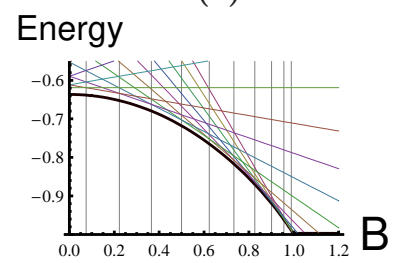

(b)

\section{Concurrence}

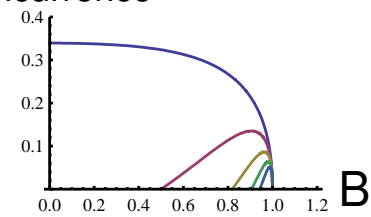

FIG. 2: (color online) (a) The ground state energy per spin (black curve) against the magnetic field $B$ in the thermodynamic limit. The energy for $N=20$ is also plotted, together with the intersections of the eigenvalues occurring at the crossing points $B_{k}=\cos [k \pi /(N+1)]$ (vertical lines). (b) Thermodynamic limit concurrence between two spins at distance $r=1$ (blue), $r=2$ (red), $r=3$ (yellow), $r=4$ (green) and $r=5$ (cobalt). Entanglement between any two spins decreases as the distance between the spins increases. It is notable that all the parameters in the figures are dimensionless.

which is analytic everywhere within the critical region, except for $B= \pm 1$. From the finite size analysis, however, we know that such region consists of dense set of crossings points (see Fig. 2 (a)) and therefore can be considered as a line of continuous QPT, with the ground state driven by $B$ through various symmetric spin subspaces with $k \approx(N+1)(\arccos B) / \pi$ flipped spins. The correlation functions behave differently for spins close to the chain boundaries and for bulk spins. Setting the distance $r=m-l$, the limit $N \rightarrow \infty$ leads to different behaviors of $\left\langle\sigma_{l}^{\alpha} \sigma_{l+r}^{\alpha}\right\rangle$ depending on whether $l \gg r$ (bulk spins) or $l \ll r$ (end point spins), 14]. In particular, for $B=0(\omega=\pi / 2),\left\langle\sigma_{l}^{z} \sigma_{l+r}^{z}\right\rangle=\left(1 / r^{2}\right)(l+r)^{2} /(l+r / 2)^{2}$, with a long distance behavior independent of boundary conditions. In contrast, longitudinal correlations are sensitive to the boundary

$$
\left\langle\sigma_{l}^{x} \sigma_{l+r}^{x}\right\rangle=\left\{\begin{array}{cc}
\sqrt{2} \tilde{A}^{2} r^{-1 / 2} & l \gg r \\
4 K\left(\frac{l+1}{2}\right)(\sqrt{2}) \tilde{A}^{2} r^{-3 / 4} & l \ll r
\end{array}\right.
$$

where $\tilde{A}=0.6450025$ and $K(x)$ is a function given in [14].

For $N \rightarrow \infty$, we find $g_{l, l+r} \simeq 2 / \pi[\sin \omega r / r-\sin \omega(2 l+$ $r) /(2 l+r)]$, so that, for bulk spins at $B=0,\left\langle\sigma_{l}^{x} \sigma_{l+r}^{x}\right\rangle=$ $\left(\frac{2}{\pi}\right)^{r} \prod_{j=1}^{[r / 2]-1}\left(\frac{4 j^{2}}{4 j^{2}-1}\right)^{r-2 j}$ where $[x]$ is the closest integer larger than $x$, and $\left\langle\sigma_{l}^{z} \sigma_{l+r}^{z}\right\rangle=\left[1-(-1)^{r}\right] \frac{(-2)}{\pi^{2} r^{2}}$, which agrees with [15].

The concurrence $C_{l, l+r}$ can be derived from the above correlation functions. We find that $\lim _{l \rightarrow \infty} C_{l, l+r}$ disappears for $r \geq 2$. Only two nearest spins are entangled, with $\lim _{l \rightarrow \infty} C_{l, l+1}=0.339$ at $B=0[16$. Bulk concurrence $\lim _{l \rightarrow \infty} C_{l, l+r}$ between two spins at distance $1 \leq r \leq 5$ is plotted for various values of $B$ in Fig. 2 (b), where the decay of entanglement with the distance is also shown. For nearest neighboring spins, the concurrence goes $\sim 1 / B$, disappearing at $B=1$. Near this point, bipartite entanglement appears for every $r$ and any 
two spins in the chain become entangled, although the magnitudes become smaller and smaller with $r$. This is because $B=1$ is the factorizing point for the XX model, with diverging entanglement range [17]. The one-tangle $\lim _{l \rightarrow \infty} \tau_{l}$ behaves in a similar way.

\section{DISCUSSIONS}

In this section we show how the ground state instability and the edge entanglement are strictly related to the emergence of quasi long range order. It is convenient to employ a dual basis to describe the system: $\mu_{n}=\prod_{m \leq n} \sigma_{m}^{x}$. Once applied to a fully polarized state, $\mu_{n}$ creates a topological excitation (a kink) [6, 18]. Indeed, any state with $k$ spin flips (i.e., after $k$ crossings) can be viewed as suitable combination of $k$ kink-antikink pairs. When $B>1$, there are no kinks and the state is separable. Near the critical point, with $B=1-\epsilon$, the ground state consists of a superposition of states with a single spin-flip, or a sea of condensed kink-antikink pairs (of infinite length for $N \rightarrow \infty$ ); such a condensation gives rise to the divergence of the concurrence range. By decreasing $B$, the size of the kink-antikink pairs decreases and their number increases. At $B=0$, the ground state has a single (degenerate) kink, with half of the spins pointing down and half pointing up. The state is 'highly' symmetric and every spin is maximally entangled with the rest of the chain, but bipartite entanglement is present with the nearest neighbor only. This is due to the fact that the concurrence depends (and it is always smaller than) the longitudinal correlation function, which ultimately tends to zero because of the presence of the kinks. Indeed, with quasi-long range order (which arises because the spinwaves are massless), the long range correlation function decays since the kinks are heavy. The critical region is an instability line because the system is driven through different Hilbert space sectors labelled by different quantum numbers (the eigenvalues of the total magnetization $\sum_{i} \sigma_{i}^{z}$ ) with different number of kinks-antikinks pairs. At finite size the phenomenon of switching among states with a different number of kinks is witnessed by the sequence of jumps in the entanglement, which are smeared out for $N \rightarrow \infty$, except at $|B|=1$ as evidenced by the partial state fidelity.

Kinks are essentially bulk excitations and the picture above is modified near the boundaries. Indeed, surface spins share entanglement differently from bulk ones: for small magnetic fields, every spin is highly entangled with the rest, but the end-spins participate essentially to bipartite entanglement, while the bulk ones are rather involved in multi-partite correlations. On the other hand, near $|B|=1$, surface spins are less entangled than those at the center (indeed, the first jumps of $\tau_{1}$ and $C_{1,2}$ are smaller than the corresponding ones for bulk spins). Thus, end spins are less sensitive to the onset of the crit- ical region (as shown also by the fidelity), while they are more entangled when the quasi long range order is fully established. This peculiar edge entanglement is reminiscent of the solitonic edge states found in [4] and could constitute a fingerprint for topological order in one dimension, valid beyond our specific model. Finally we note that preliminary analysis have shown that the formalism we have developed opens the way for the understanding the role of cluster type states [19] close to QPT, especially for quantum information purposes. Indeed, a Hamiltonian for the cluster state can be mapped onto a model with Ising order by means of the dual transformations employed above. This provides a non-trivial disentangling protocol for the cluster states [20] that will be explored in a subsequent work.

Summarizing, through the study of the entanglement content and of the fidelity of the ground state, we have investigated the quasi long range order in the XX model and its connection to the quantum instability arising at finite size as a result of the presence of topological excitations. We have also discussed the special entanglement properties of the edge spins arguing that they are a direct manifestation of the topological character of the QPT.

Acknowledgements- This work is supported by the National Research Foundation \& Ministry of Education, Singapore. V. Vedral acknowledges to EPSRC, the Royal Society and the Wolfson Foundation for financial support. We thank J. Kwon, I. Lawrie, H.-J. Mikeska, D. Patane' and A. Seel for discussions.

[1] S. Sachdev, Quantum Phase Transitions, Cambridge University Press (1999).

[2] V.L. Berezinskii, Zh. Eksp. Teor. Fiz. 59, 907 (1970) [Sov. Phys. JETP 32, 493 (1971)]; J.M. Kosterlitz and D.J. Thouless, J. Phys. C 6, 1181 (1973).

[3] M. Takahashi, Thermodynamics of one dimensional solvable models, (Cambridge Univ. press, Cambridge 1999).

[4] I. Affleck et al., Phys. Rev. Lett. 59, 799 (1987); Y. Kitaev, cond-mat/0010440.

[5] L. Amico et al., Rev. Mod. Phys. 80, 517 (2008).

[6] E. Fradkin and L. Susskind, Phys. Rev. D 17, 2637 (1978).

[7] A similar kind of instability has been found for the Dicke model in V. Bužek, M. Orszag and M. Rosko, Phys. Rev. Lett. 94, 163601 (2005).

[8] H. T. Quan et. al., Phys. Rev. Lett. 96, 140604 (2006); P. Zanardi and N. Paunkovic, Phys. Rev. E 74, 031123 (2006).

[9] S. Katsura, Phys. Rev. 127, 1508 (1962);A. De Pasquale et al., Eur. Phys. J. Special Topics 160, 127 (2008). (2008), see also

[10] E. Lieb,T. Schultz and D. Mattis, Annals of Phys. 16, 407 (1961).

[11] W. K. Wootters, Quantum Inf. Comput. 1, 27 (2001); W. K. Wootters, Phys. Rev. Lett. 80, 2245 (1998).

[12] H.-Q. Zhou, R. Orùs, and G. Vidal, Phys. Rev. Lett. 100, 
080601 (2008); Paunkovic et al, Phys. Rev. A 77, 052302 (2008); H. Kwok, C. Ho and S. Gu, quant-ph/0805.3885 ;W.-L. You, Y.-W. Li, and S.-J. Gu, Phys. Rev. E 76, 022101 (2007).

[13] G. Vidal et al., Phys. Rev. Lett. 90, 227902 (2003); J. I. Latorre, E. Rico, and A. Kitaev, Quant.Inf.Comput. 4, 48 (2004);F. Franchini et al., J.Phys. A, 40, 8467 (2007).

[14] H.-J. Mikeska and W. Pesch, Z. Phys. B 26, 351 (1977).

[15] T. Tonegawa, Solid State Comm. 40, 983 (1981).

[16] B.-Q.Jin and V.E. Korepin, Phys. Rev. A 69, 062314
(2004).

[17] L. Amico et al., Phys. Rev. A 74, 022322 (2006).

[18] H.-J. Mikeska, S. Miyashita, and G.H. Ristow, J. Phys. C 3, 2985 (1991).

[19] H. J. Briegel and R. Raussendorf, Phys. Rev. Lett. 86, 910 (2001);J. Pachos and M. Plenio, Phys. Rev. Lett. 93, 056402 (2004).

[20] M. Plenio, J. Mod. Opt. 54, 349 (2007). 